lich gelangen, glaubte ich mit Sicherheit auf das Vorhandensein von Amyloid schliessen zu dïrfen. Vorzugsweise bediente ich mich zu meinen Untersuchungen amyloider Nieren. - Darin stimmen alle Autoren überein, dass es der Gefässapparat ist, welcher vorzugsweise und namentlich zuerst erkrankt. Stets soll die Entartung an den Glomerulis beginnen und von da auf die Vasa afferentia, die Art. interlobulares, sowie auf die gestreckten Arterien des Marks übergreifen. In letzter Reihe erkranken die Vasa efferentia und die Capillaren der Rinde. Die Glomeruli bilden gewöhnlich den am meisten erkrankten Abschnitt des Gefässsystems, zuweilen den einzigen. Trotzdem möchte ich nicht zugeben, dass sie unter allen Umständen stet. zuerst erkranken; dagegen sprechen Fälle, wie ich sie untersucht habe, bei denen nur eine fleckweise Erkrankung der Glomerularschlingen vorhanden war, während die Interlobulararterien und namentlich die Art. rectae des Marks fast in toto degenerirt waren. Die degenerirten Glomeruli haben, wenn die Entartung alle oder den grössten Theil der Capillarschlingen ergriffen hat, meistens eine bedeutende Zunahme ihrer Grösse erlitten; ihre Kerne sind häufig selbst noch in solchen Fällen deutlich erkennbar, in welchen der gesammte Glomerulus auf Zusatz des Reagens die charakteristische Färbung angenommen hat. Erst wenn der ganze Gefässknäuel in eine gleichmässig glasige, schollige Masse verwandelt ist, werden die Kerne unsichtbar. An den arteriellen Stämmchen soll, wie dies allgemein angenommen wird, die Entartung stets an der Muscularis beginnen und von hier auf die anderen Gefässhäute übergehen. Ich habe mich zu wiederholten Malen davon überzeugen können, dass diese Angabe nicht für alle Fälle zutrifft. Man findet zuweilen Gefässstämmchen, über deren arterielle Natur das Vorhandensein der Musculatur keinen Zweifel lassen kann, mit fleckweise erkrankter Adventitia bei ganz normaler Muscularis.

Zuweilen gelingt es, den Nachweis zu führen, dass die Erkran-

\section{Ueber Amyloiddegeneration.}

\section{Von Prof. Dr. M. Litten.}

(Fortsetzung ans No. 24.)

Sie sehen also, dass er angiebt, dass Lebern, wenn sie schon vollständig steif und transparent geworden sind, noch functionsfähig bleiben können, eine Beobachtung, welche ich ebenfalls (l. c.) wiederholt gemacht habe. Was von der Leber gilt, gilt in noch höherem Grade von der Niere und gelegentlich auch vom Dünndarm. Ich habe sehr weit gehende Entartung desselben gesehen, ohne dass je Durchfälle aufgetreten wären. Schon daraus geht hervor, dass die amyloide Degeneration keineswegs jedesmal, wo sie auftritt, die Gefässe, oder allgemeiner ausgedrückt, die erkrankten Gewebstheile in eine structurlose Masse verwandelt, welche des Organischen entkleidet und für die physiologischen Functionen untauglich ist. Wir werden vielmehr, wie bei allen übrigen Krankheiten mit progressiver Tendenz, auch hier alle Stadien der amyl. Degeneration und alle Uebergänge von der eben erkennbaren Infiltration des Gewebes bis zum vollständigen Untergang desselben und bis zur totalen Umwandlung in jene structurlose, nicht mehr functionirende Masse, welche wir als Amyloid bezeichnen, neben einander vorfinden. Dass dies in der That der Fall ist, lehrt nicht nur die klinische, sondern in noch viel höherem Grade die anatomische Untersuchung.

Um dies zu beweisen, muss ich auf die zahllosen Untersuchungen eingehen, welche ich an amyloiden Organen, vorzugsweise Nieren, angestellt habe, um zu zeigen, in welcher Weise die Gefässe von der amyloiden Degeneration ergriffen werden. Denn es kommt mir darauf an, die Frage zu erörtern, ob in den Fällen von Syphilis und Caries, bei welchen in Folge der Amyloiddegeneration der Nieren Eiweiss ausgeschieden wird und bei beginnender Heilung des Grundleidens wieder verschwindet, eine Auflösung und Fortschaffung d.h. eine Resorption der in der Gefässwand abgelagerten amyloiden Substanz stattfindet. Wahrscheinlich beruht der Anfangsprocess der amyloiden Umwandlung der Gefässwände auf einer Art Gerinnung, ähnlich der des Fibrins, wobei, wie die anatomische Untersuchung zeigt, der Process stets ron ganz kleinen Heerden in der Muscularis ausgeht. Diese letzteren, nicht grosse, in toto amyloid degenerirte Gewebspartien habe ich im Auge, wenn ich die Möglichkeit der Resorptionsfähigkeit amyloider Heerde discutire. Denn wo überhaupt klinisch die Möglichkeit einer relativen Heilung in Frage kommt, muss der allgemeine Process nicht nur zum Stillstand gelangen, sondern es müssen auch diejenigen Veränderungen, auf denen die Eiweissausscheidung beruhte, einer Rückbildung im Sinne einer Heilung, unterliegen, damit eben die Albuminurie aufhören kann. Welcher Art diese Rückbildung ist, werden wir später bei Besprechung der einschlägigen Versuche discutiren; vorher noch einige Worte über die anatomischen Veränderungen selbst.

Zur Ausführung der Amyloidreaction bediente ich mich ausschliesslich der älteren; von Virchow eingeführten Methode (Jodjodkaliumlösung mit und ohne Zusatz von $\mathrm{SO}_{3}$ ) sowie der Anilinfarbstoffe (vorzugsweise des Methylvioletts); nur da, wo beide Reactionen deut- kung von der Intima ausgegangen ist; alsdann sieht man auf Querschnitten, die z. B. mit Methylviolett behandelt sind, rothgefärbte Herde in der Intima, während die Media durchweg blan-violett gefärbt erscheint. Entsprechend verhalten sich die Dinge bei Jodbehandlung. In der überwiegenden Mehrzahl der Fälle jedoch beginnt die Erkrankung an der Media, geht aber bei nur einigermassen ausgedehnter Entartung sehr bald auf die übrigen Gefässhäute über, so dass bald die Gefässwand in ihrer ganzen Dicke infiltrirt ist. Anfangs gelingt es alsdann noch, die Structur derselben zu erkennen und die einzelnen Häute $\mathrm{zu}$ differenziren, bald indess bei fortschreitender Degeneration ist dies nicht mehr möglich, und das ganze Gefüssrohr ist in eine einförmige structurlose und durchsichtige Masse verwandelt. Der Grad und die Ausdehnung der Entartung an den einzelnen Gefässen ist äusserst ungleichmässig. Vergleichende Untersuchungen an möglichst umfangreichen Längsund Querschnitten ergaben, dass die amyloide Infiltration sich weder immer von einem Anfangsherd der Continuität nach ausbreitet, noch an einer erkrankten Stelle stets die ganze Wanddicke des Gefässes ergreift. So erhält man z. B. an glücklich getroffenen Längsschnitten folgendes Bild : man sieht eine Art. interlobularis mit einem oder mehreren abgehenden Vas. affer. und den dazu gehörigen Glomerulis. Die Reaction mit Methylviolett zeigt die erstgenannte Arterie an irgend einer Stelle ihres Verlaufes in ganzer Wanddicke erkrankt, nun folgt ein anderer Abschnitt, welcher absolut gesund erscheint, und dann ein dritter, dem Vas. afferens zunächst gelegener, welcher wieder prachtvoll roth gefärbt ist. Ein zunächst abgehendes Vas afferens ist vollständig intact bis dicht vor seiner Auflösung in die Schlingen des Glomerulus; erst hier beginnt die amyloide Entartung, welche sich in verschiedener Intensität auf denselben fortsetzt. Daneben sieht man ein anderes Vas afferens, welches in der Mitte seines Verlaufs an irgend einer Stelle die charakteristische Reaction zeigt, während Anfangs- und Endtheil gesund erscheinen, und dessen dazu gehöriger Glomerulus die rothe Farbe in voller Pracht erkennen lässt. Dass unter solchen Umständen von einer Erkrankung per continuitatem keine Rede sein kann, liegt auf der Hand. Vielmehr muss man annehmen, dass die Degeneration gleichzeitio an mehreren Stellen ein und desselben Gefässrohres resp. desselben Gefässsystems (d. h. in dem Verästlungsgebiet einer Arterie) ihren Anfang nehmen kann. Ebensowenig gleichmässig wie in Bezug auf die Continuität des Gefässrohrs entwickelt sich die amyloid. Degeneration auch in Bezug auf den Querschnitt desselben. Weder finden wir die Gefässe stets in ihrer ganzen Circumferenz erkrankt, noch umfasst die amyloid. Infiltration stets alle Gefässhäute in gleicher Ausdehnung. Wenn man eine grosse Reihe von gut getroffenen, amyloid entarteten Gefässquerschnittenuntersucht, so findet man eine Anzahl, welche das eben Gesagte in deutlicher Weise demonstriren.

Während viele Querschnitte, auf welche man Methylviolett einwirken lässt, einen gleichmässig breiten, überall geschlossenen rothen Ring darstellen, findet man andere, bei welchen der Ring nicht vollständig geschlossen ist, sondern an irgend einer Stelle 
durch eine blaue oder mehr violett gefärbte Zone der Gefässwand unterbrochen erscheint. In anderen Fällen erkennt man in dem durchweg blau gefärbten Querschnitt in verschiedener Anzahl kleine rothe Einlagerungen, welche eben den ersten Beginn der amyl. Infiltration darstellen. Häufig ist es ferner ausschliesslich die Muscularis, welche in der ganzen Circumferenz erkrankt ist, so dass man dieselbe auf dem Querschnitt als rothen Kranz sieht, während die Adventitia und Intima intact sind. Gewöhnlich betheiligt sich auch die Adventitia sehr früh an der Entartung, so dass die Intima allein blau gefärbt erscheint. Allmählich wird auch sie infiltrirt, und dann sieht man den rothen Ring je nach der Wandstärke in verschiedener Dicke. Im Centrum erkennt man das kreisrunde Lumen von anfangs normaler Weite, oder später bei zunehmender Infiltration der Intima als kaum noch erkennbaren Kreis. Schreitet der Process in seiner Entwicklung weniger regelmässig vor, so kommt es zu ungleichmässiger Infiltration der einzelnen Häute, vorzugsweise der Muscularis. Hierdurch können circumscripte Ausbuchtungen der Gefässwand, namentlich nach dem Lumen zu erfolgen, welche eine Verzerrung und in höheren Graden eine zuweilen sehr bedeutende Verengerung des letzteren bedingen. Bei zunehmender Entartung, und namentlich bei gleichmässiger Infiltration werden die Lumina immer enger, bis schliesslich das Gefässrohr vollständig undurchgängig erscheint; dass auch in solchen Fällen indess häufig noch eine Injection möglich ist, davon habe ich mich bei meinen Injectionsversuchen oft genug überzengt. Ich bediente mich zur Ausführung der letzteren stets einer dünnen, die Capillaren durchdringenden Leimlösung, welche mit Berliner Blau gefärbt war. Diese Injectionsmasse wurde warm in das erwärmte intacte Organ theils mit der Hand, theils unter constantem Druck von $100-120 \mathrm{~mm} \mathrm{Hg}$ injicirt. Die Nieren wurden alsdann erhärtet und nach vorangegangener Färbung mikroskopisch untersucht. An Präparaten, welche auf diese Weise gewonnen waren, erkannte man sehr leicht, dass auch intensiv entartete Gefässe für die Injectionsmasse noch sehr wohl durchgängig sein können. Selbst wenn ein arterielles Gefäss an einer Stelle so verengt erschien, dass auf dem Längsschnitt die bis dahin breite Säule der blauen Injectionsmasse bis auf einen ganz schmalen Streif reducirt war, sah man peripher von dieser Verengerung, sobald die Lichtung des Gefässes wieder zunahm, die letztere wieder vollständig mit der Farbmasse erfüllt. Von diesem Vorkommen kann man sich namentlich an den Rindengefässen sehr gut überzeugen; hier sieht man z. B. eine Art. interlobularis, welche an einer Stelle fast bis zum Verschwinden ihres Lumen verengt erscheint; unmittelbar jenseits der Stenose ist sie wieder vollständig injicirt, und ebenso erscheinen die peripher von jener Stelle abgehenden Vasa afferentia in toto injicirt; oder: ein Vas afferens zeigt in seinem Verlauf eine derartige Verengerung, und trotzdem ist nicht nur der peripher davon gelegene Abschnitt, sondern auch der zugehörige Glomerulus vollständig injicirt. Dass es sich hierbei nicht um Füllung auf collateralem Wege gehandelt haben kann, liegt auf der Hand, denn für den Glomerulus ist sein zuführendes Vas afferens stets ein Endgefäss. Die eben erwähnten Stenosen im Verlauf einer amyloid erkrankten Arterie findet man gar nicht so selten; sie sind der Ausdruck jener bereits erwähnten ungleichmässigen Entartung der Muscularis, welche spindelartige Anschwellungen bildet, wodurch das Lumen verengt wird. Aber ganz abgesehen von diesen circumscripten Gefässstricturen erwachsen dem Blutstrom durch die gleichmässige amyl. Infiltration der Gefässwände bedeutende Hindernisse. Wenn diese durch die künstliche Injection auch häufig in vollständiger Weise überwunden werden, und selbst intensiv entartete $\mathrm{Ge}-$ fässe möglichst vollständig injicirt erscheinen, so darf man selbstverständlich hieraus keine Rückschlüsse auf die Circulation intra vitam machen. Dasselbe, was für die Arterien gilt, gilt auch für die Capillaren der Glomeruli. Diese können bei starker Entartung für die Injection absolut undurchgängig werden, wie in dem zuerst mitgetheilten Fall. Man sieht alsdann den purpurrothen homogenen Gefässknäuel ohne jede Spur blauer Injectionsmasse. In allen anderen Fällen fanden sich gänzlich uninjicirte Glomeruli nur ganz vereinzelt, während alle übrigen theilweise injicirt waren, und zwar um so vollständiger, je weniger entartet sie waren. Häufig bekommt man Bilder, wobei in einem und demselben Glomerulus einige Schlingen injicirt sind, andere amyloid entartete dagegen keine Injectionsmasse aufgenommen haben. In noch anderen erkennt man die Injectionsmasse auch in amyloid entarteten Schlingen als blaue Streifchen oder Klümpchen.

Als Nebenbemerkung möchte ich hier noch einschalten, dass in keinem der vielen Fälle, in welchen ich die Niere injicirt hatte, durch die Injection Extravasate entstanden waren, sodass man eine vermehrte Brüchigkeit der amyloiden Gefässe nicht wohl annehmen darf. Hiermit scheint nun auch die klinische Thatsache in Uebereinstimmung zu stehen, dass man bei der amyl. Nierenentartung viel seltener Haemorrhagien (auf der Haut, in der Retina, im
Gehirn etc.) auftreten sieht, als bei den anderen Formen der Nierenerkrankung, namentlich den entzündlichen.

Sie sehen also daraus, meine Herren, dass es sich um structurlose Massen keineswegs in der Mehrzahl der Fälle handelt; es handelt sich vielmehr in den meisten Fällen - wenn man nicht gerade die allerfortgeschrittensten zu Gesicht bekommt - um kleinere Processe, welche an einzelnen Stellen nicht einmal die ganze Wanddicke der Gefässe, und diese auch nicht einmal in grosser Erstreckung einnehmen.

Die Frage, um welche es sich nun zunächst handelte, und welche ich durch einen einfachen Versuch ihrer Lösung näher zu führen hoffte, war die: auf welchem Process beruht das Aufhören der Albuminurie in leichten Fällen von amyloider Degeneration der Nieren? Denn dass es sich nur um diese handeln könne, bei welchen die Erkrankung nur in ihren ersten Anfängen aufgetreten, und

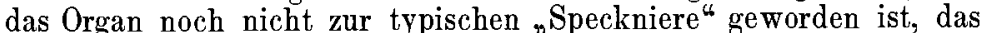
liegt auf der Hand. Hätten wir es nur mit einem Stillstand der sonst progressiv fortschreitenden Entartung zu thun, so würde da. durch allein das Aufhören der Albuminurie noch nicht genügend erklärt sein. Entweder müsste sich eine Rückbildung der erkrankten Gefässe im Sinue einer Heilnng vollziehen, denn eine andere Ursache für die Albuminurie ist bei der "rein en "Form der amyloiden Degeneration bis jetzt nicht erwiesen, oder man müsste die Ursache für das Aufhören der Eiweissauscheidung in günstigeren Bedingungen des Blutdrucks und Beschleunigung der Circulation suchen. Schon vor langer Zeit habe ich den Nachweis zu führen versucht, dass die Verlangsamung der Circulation in der Niere allein genügt, um Eiweiss im Urin auftreten zu machen (cf. Ctblatt f. d. med. Wiss.) Ungleich schwieriger ist es, sich eine Vorstellung darüber zu bilden, was aus der amyloiden Gefässwand im Fall einer Rückbildung wird. Handelt es sich im Beginn der Erkrankung nur um eine Einlagerung (Infiltration) in dieselbe, ohne dass die erkrankte Cefässwand selbst in amyloide Substanz übergeht, - und zu diesem Schluss bin ich durch die Untersuchung eben erkrankter Gefässe gekummen -, so ist die Möglichkeit einer Auflösung und Resorption nicht von der Hand zu weisen. Um das Schicksal amyloider Substanz in möglichst objectiver Weise zu verfolgen, schien es mir am passendsten, dieselbe in die Bauchhöhle von Thieren zu implantiren und längere Zeit darin zu belassen.

Ich nahm nun die degenerirten Organe, namentlich Nieren, schnitt sie in ganz kleine Würfelchen von der Grösse eines grösseren Stecknadelknopfes und brachte sie Thieren in die Bauchhöhle ein. Ich will hier nicht auf meine Vorversuche eingehen, sondern gleich die Resultate mittheilen. In denjenigen Fällen, in welchen ich so total degenerirte Nieren nahm, dass das ganze Gesichtsfeld des Schnittes bei der Behandlung mit Methylviolett in ein leuchtendes Roth verwandelt wurde, wo also das ganze Gewebe in toto eine einzige amyloide Masse darzustellen schien, bekam ich allein positive Resultate. Nahm ich weniger hochgradig entartete Organe, so war später die Vergleichung mit dem ursprünglichen Organ zu schwierig. Wenn nun solche kleine Würfel von total amyloiden Organen 4,5 bis 6 Monate in der Bauchhöhle der Thiere verweilt hatten, öffnete ich dieselbe und suchte die implantirten Stückchen wieder herauszubekommen. Ich fand sie in kleinen Bindegewebssäckchen liegend, welche meist am freien Rande des Mesenteriums angewachsen waren und eine äusserst reichliche Vascularisation erkennen liessen, wobei Arterienstämmchen in grosser Anzahl von dem Mesenterium zu den Säckchen herüberzogen. Holte ich nun die kleinen Partikelchen heraus, so erschienen dieselben für das blosse Auge als vollkommen porös, wie Elfenbeinzapfen, die man in Knochen eingeführt hat. Die Fremdkörper-Partikel, welche also eine unebene Oberfläche hatten und wie angenagt aussahen, liessen in ihrem Innern bei der mikroskopischen Untersuchung eine grosse Menge Riesenzellen erkennen, in denen vielfach kleinste Theilchen der Fremdkörper eingeschlossen waren, so fanden sich z. B. wiederholt als Inhalt der Riesenzellen amyloide Glomeruli. Wahrscheinlich handelte es sich dabei um eingewanderte Leukocyten, welche sich, wie in den Versuchen von Ziegler, in Riesenzellen umgewandelt und dabei Partikel der Niere in sich aufgenommen hatten. Bei der weitern Untersuchung der implantirten Stückchen, welche alle von demselben amyloiden Organ stammten, das ich natürlich zum Vergleich aufgehoben hatte, stellte sich nun Folgendes heraus: In den seltensten Fällen nur war, wenn ich mit Methylviolett reagirte, eine so leuchtende Rothfärbung zu erkennen, wie an der ursprünglichen Niere. Dagegen fanden sich an den verschiedenen Versuchspartikelchen alle möglichen Uebergangsfarben vom tiefsten Burgunderroth bis zum hellen Rosa, ja bis zu einer Nuance, welche zwischen Roth und Blau in der Mitte stand, so dass man nicht mehr bestimmt sagen konnte, ob hier noch eine bestimmte Methylviolettreaction vorhanden war oder nicht. Die letzteren Partikelchen reagirten auf Jod nicht mehr, dagegen boten sie alle chemischen und physikalischen Eigenschaften 
des Amyloids dar, d. h. sie waren transparent, homogen, stark lichtbrechend, nicht in Alkohol, Kalilauge, Säuren etc. löslich. Betreffs der Farbenreaction kann man im Allgemeinen sagen, dass die Jod- und Jod-S0 ${ }^{3}$-Reaction nur da gelang, wo auch gleichzeitig eine ausgesprochene Rothfärbung bei Anwendung von Methylviolett eintrat. Wo die letztere nicht deutlich gelang, blieb auch stets die Jodreaction aus; die Substanz färbte sich alsdann gelblichbraun.

Es scheint aus diesen Versuchen sich das Resultat zu ergeben, dass unter geeigneten Verhältnissen, welche wahrscheinlich in der reichlichen Vascularisation zu suchen sind, aus dem reinen Amyloid eine Modification gebildet wird, welche alle chemischen und physikalischen Eigenschaften der amyloiden Substanz hat, ohne die charakteristischen Färbeeigenthümlichkeiten derselben $\mathfrak{z u}$ besitzen.

Dass diese Thatsache eine gewisse Bedeutung verdient, das glaube ich, werden Sie mir zugeben, wenn ich Ihnen zunächst eine andere rein histologische Thatsache mittheile. Bei den vielen Untersuchungen, die ich an den verschiedensten amyloiden Organen ausgeführt habe, ist mir seit Jahren ein merkwürdiger Unterschied in der Färbbarkeit der amyloiden Substanz bei Anwendung der Anilinfarben aufgefallen. Bei allen in der letzten Zeit eingeführten Theerfarben, namentlich dem Jodgrün von Stilling oder dem von Curschmann eingefïhrten Methylgrün, sowie dem lange gebrauchten Methylviolett, welche alle drei die Eigenschaft haben, das normale Gewebe blau zu färben, während die amyloiden Theile roth werden, ist mir aufgefallen, dass die Rothfärbung der Amyloidsubstanz, selbst in Fällen unzweifelhafter positiver Reaction bei Anwendung von Jodjodkalium mit und ohne Zusatz von $\mathrm{SO}_{3}$, keineswegs stets dieselbe ist, sondern vielfach differirt und alle denkbaren Farbennuancen des ausgesprochenen Roth's darbietet, vom leuchtendsten Burgunderroth bis zu den helleren Nuancen. Andere Male findet man auch Fälle, bei welchen Organabschnitte die genau die physikalischen und chemischen Eigenschaften der amyloiden Substanz darbieten, d. h. denselben Glanz, dieselbe Transparenz, dasselbe Lichtbrechungsvermögen, dieselben Unlöslichkeitsverhältnisse gegenüber Säuren und Alkalien, ohne dass die Farbenreaction gegenüber Jod und $\mathrm{Jod}_{-}-\mathrm{SO}_{3}$, sowie gegenüber den Theerfarben ein positives Resultat ergiebt. 